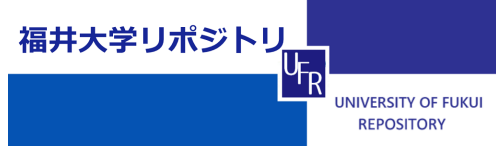

Desi gn of a quasi - opt i cal mode conver si on syst em wi th variable out put beam si ze

\begin{tabular}{|c|c|}
\hline 著者 & OGAMA I, I DEHARA T, KASPAREK W \\
\hline $\begin{array}{l}\mathrm{j} \text { our nal or } \\
\text { publ i cat i on } \mathrm{titl} \text { e }\end{array}$ & I nt er nat i onal J our nal of El ect r oni cs \\
\hline vol une & 87 \\
\hline nunber & 4 \\
\hline page $r$ ange & 457- 467 \\
\hline year & $2000-04$ \\
\hline URL & ht t p: //hdl . handl e. net /10098/1520 \\
\hline
\end{tabular}




\title{
Design of a quasi-optical mode conversion system with variable output beam size
}

\author{
I. OGAWA, T. IDEHARA and W. KASPAREK
}

The quasi-optical system consisting of a quasi-optical antenna and two parabolic mirrors can convert the TE 03 mode output $(f=301 \mathrm{GHz})$ of the Gyrotron FU IV into a well collimated, linearly polarized beam. This system offers the possibility of tuning the size of the beam produced from $5 \mathrm{~mm}$ up to $24 \mathrm{~mm}$ by rotating and moving the two parabolic mirrors.

\section{Introduction}

Electromagnetic waves in the submillimetre wavelength range are used for developments in numerous fields including material physics, plasma diagnostics, astronomy, biophysics and material processing. Some applications such as plasma scattering measurements (for example Fekete et al. 1994, Terumichi et al. 1984, Suvorov et al. 1997) need more intense waves.

Molecular vapour lasers (Semet et al. 1980, Kawahata et al. 1988) and backwardwave oscillators (Morino et al. 1997) have been used as a power source in this wavelength range because they produce stable continuous wave (CW) outputs as Gaussian beams. On the other hand, high frequency gyrotrons are the most promising candidates for delivering intense waves of several hundred watts up to several kilowatts (Zaytsev et al. 1974, Spira-Hakkarainen et al. 1990, Idehara et al. 1995). In addition, CW operation has been achieved with high frequency gyrotrons (Hong et al. 1993, Idehara et al. 1998).

The gyrotron output should be converted into a well collimated, linearly polarized beam because the gyrotron delivers the $\mathrm{TE}_{m n}$ waveguide mode output, which is far from what is usually required as radiation power source.

Some applications of gyrotrons such as the effective coupling of beams to hybrid modes in corrugated waveguides (for example, Ohkubo et al. 1997) require matching of the size of the beam produced.

A quasi-optical antenna (Vlasov and Orlova 1974) converts the gyrotron output into a linearly polarized beam whose far-field consists of a main beam with an elliptical cross-section and additional sidelobes. As can be seen from Gaussian optics, the main beam can be converted into a circular beam by two-dimensionally adjusting the spotsize and the curvature radius of the wave front of the beam. This conversion is attained by the combination of a parabolic cylinder and a modified 
mirror similar to an ellipse or the combination of two parabolic cylinders (Ogawa et al. 1999 a). The former is suitable for getting a high quality beam, while the latter is suitable for adjusting the beam waist size because a parabolic cylinder offers the tunability of focal lengths via the variation of the angle of incidence.

As an example of this approach, the design of the quasi-optical system for the $\mathrm{CW} \mathrm{TE} \mathrm{T}_{03}$ mode output $(f=301 \mathrm{GHz}$ ) of the Gyrotron FU IV (Idehara et al. 1998) is presented.

\section{Treatment of the bean by using Gaussian optics}

The first element in the quasi-optical transmission line is the quasi-optical antenna (figure 1) consisting of a circular waveguide (internal radius $a_{w}=14 \mathrm{~mm}$ ) with a step-cut and a cylindrical parabolic reflector (focal length $f_{\mathrm{p}}=21.75 \mathrm{~mm}$ ). The antenna converts the gyrotron output $\left(\mathrm{TE}_{03}\right.$ mode, $f=301 \mathrm{GHz}$, Brillouin angle $\alpha=6.62^{\circ}$ ) into a linearly polarized beam. The electric and magnetic fields are parallel to the $x$ - and $y$-directions, respectively.

Radiation reflected from the parabolic reflector of the quasi-optical antenna is treated as if it came from a plane image source lying behind the reflector (Wada and Nakajima 1986, Brand et al. 1990).

In the design of the system (figure 2), the image source is located thus that the beam with polarization in the $x$-direction propagates along the $z$-axis. The profiles of the beam produced by the image source are calculated using the Huygens equation (Ogawa et al. 1997).

Mirror $m 1$ focuses the beam in the $y^{\prime}$-direction and mirror $m 2$ focuses the beam in the $x^{\prime \prime}$-direction. The beam produced at mirror ml contains sidelobes in addition to the main lobe, as can be seen in figure 3. In order to truncate the sidelobes, the size of the rectangular mirror $\mathrm{ml}$ is chosen to be $179 \mathrm{~mm}$ in the $x$-direction and $240 \mathrm{~mm}$ in the $y$-direction. This is possible, as for the application planned the transmission efficiency is of minor importance, whereas beam quality is a strong requirement. On the other hand, mirror $\mathrm{m} 2$ is wide enough to avoid any diffraction loss due to beam truncation.

The distance between the image source and the mirror $\mathrm{ml}$ is large $(7000 \mathrm{~mm})$ enough to guarantee that the spot sizes of the main beam are accurately given by assuming a bi-Gaussian beam whose waist $\left(w_{0 x}=38.1 \mathrm{~mm}\right.$ in the $x$-direction, $w_{0 y}=25.3 \mathrm{~mm}$ in the $y$-direction) is located at the centre of the image source.
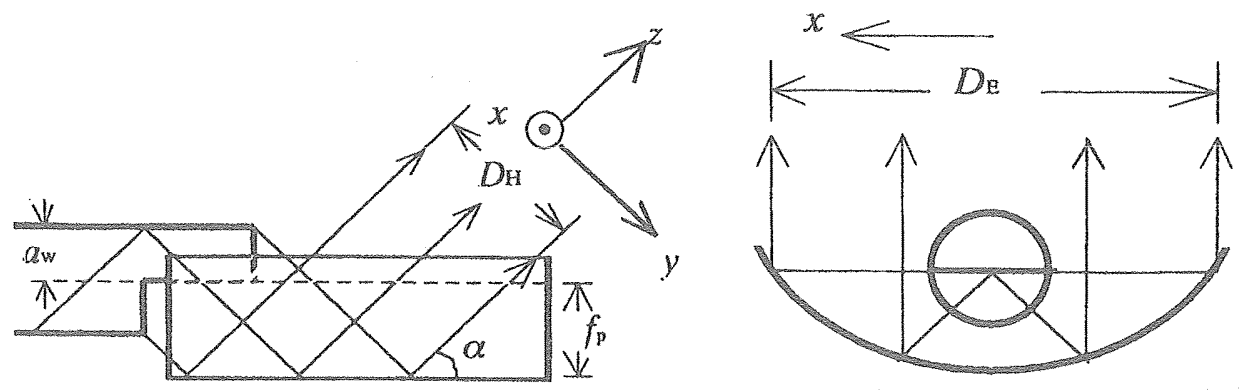

Figure 1. Quasi-optical antenna. The $x$ - and $y$-directions correspond to the directions of electric and magnetic fields, respectively. 


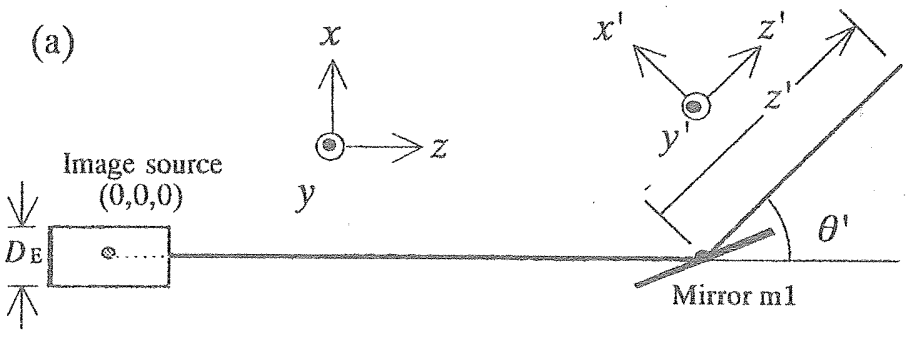

(b)

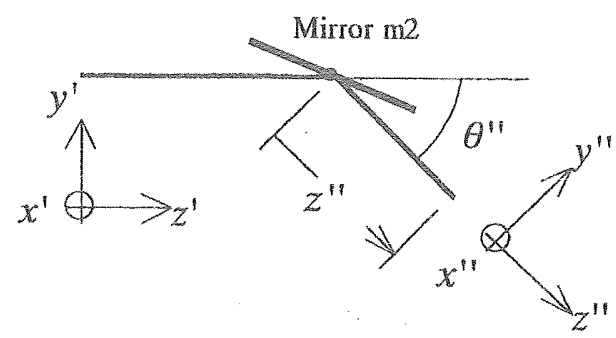

Figure 2. Quasi-optical system. (a) A plane image source is used for the calculation of the subsequent radiation patterns of the quasi-optical antenna. The mirror $m !$ focuses the beam in the $y^{\prime}$-direction. (b) The mirror $m 2$ focuses the beam in the $x^{\prime \prime}$-direction.

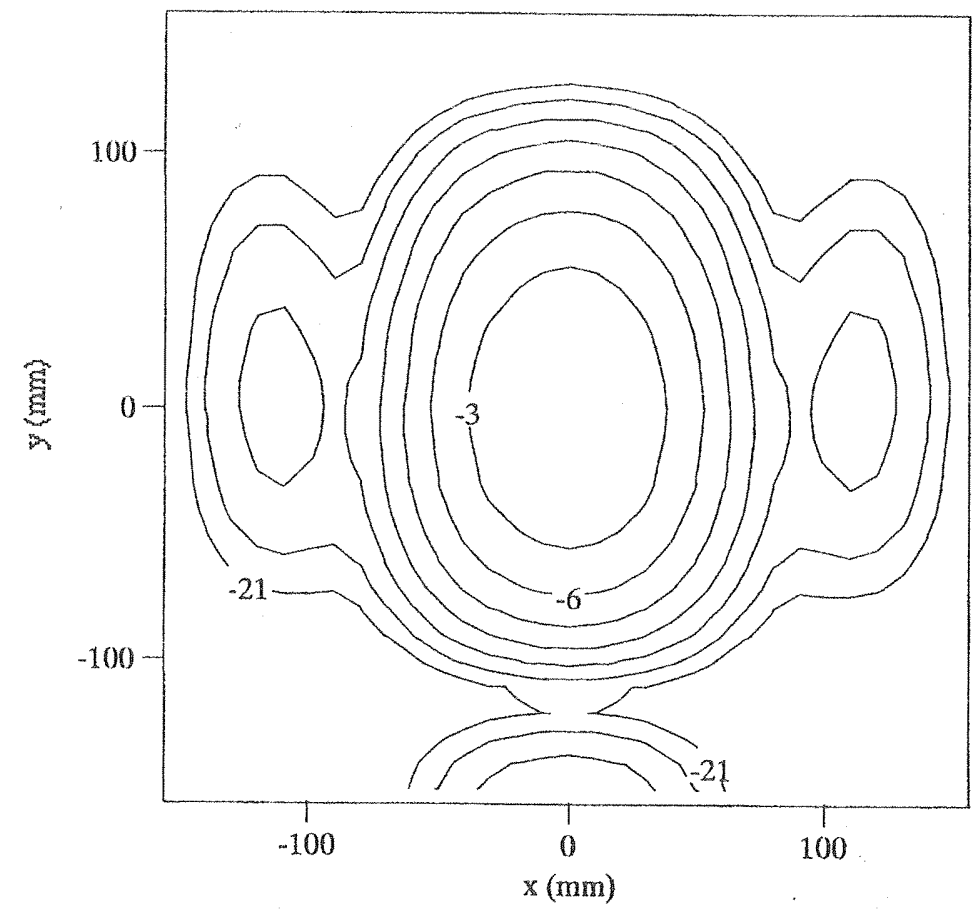

Figure 3. Calculated intensity contours at the mirror $\mathrm{ml}$. Contours are in decibels relative to the intensity maximum. 
The intensity of the bi-Gaussian beam is given by

$$
I=\frac{2 P_{0}}{\pi w_{x} w_{y}} \exp \left(-\frac{2 x^{2}}{w_{x}^{2}}\right) \exp \left(-\frac{2 y^{2}}{w_{y}^{2}}\right)
$$

where $w_{x}$ and $w_{y}$ are the spot sizes of the bi-Gaussian beam in the $x$ - and $y$-directions, respectively and $P_{0}$ is the total beam power.

The complex beam parameters $q_{x}$ and $q_{y}$ in the $x$-and $y$-directions defined by

$$
\left.\begin{array}{l}
\frac{1}{q_{x}}=\frac{1}{R_{x}}-\mathrm{j} \frac{\lambda}{\pi w_{x}^{2}} \\
\frac{1}{q_{y}}=\frac{1}{R_{y}}-\mathrm{j} \frac{\lambda}{\pi w_{y}^{2}}
\end{array}\right\}
$$

are convenient parameters to treat the beam propagation and the focusing due to the focusing element (Ogawa et al. $1999 \mathrm{~b}$ ), where $R_{x}$ and $R_{y}$ are the curvature radii of the wave fronts in the $x$ - and $y$-directions, respectively.

At the beam waist, $R_{x}, R_{y} \rightarrow \infty$. Therefore, in this case the complex beam parameters are

$$
\left.\begin{array}{l}
q_{0 x}=\mathrm{j} \frac{\pi w_{0 x}^{2}}{\lambda} \\
q_{0 y}=\mathrm{j} \frac{\pi w_{0 y}^{2}}{\lambda}
\end{array}\right\} .
$$

The complex beam parameters change, when the beam propagates or it is focused by any element. The complex beam parameters $q_{x}$ and $q_{y}$ change to

$$
\left.\begin{array}{l}
q_{x}^{\prime}=q_{x}+d \\
q_{y}^{\prime}=q_{y}+d
\end{array}\right\}
$$

after propagating a distance $d$.

After a focusing element, they change to new values given by

$$
\left.\begin{array}{c}
\frac{1}{q_{x}^{l}}=\frac{1}{q_{x}}-\frac{1}{f_{x}} \\
\frac{1}{q_{y}^{\prime}}=\frac{1}{q_{y}}-\frac{1}{f_{y}}
\end{array}\right\}
$$

where $f_{x}$ and $f_{y}$ are the focal lengths of the focusing element in both directions.

\section{Design of the system asing Garssiann optics}

To demonstrate the method, we have made a design of the system (figure 2) to produce a well collimated beam with a circular cross-section (waist size of $\left.w_{0 x^{\prime \prime}}=w_{0 y^{\prime \prime}}=w_{0}^{\prime \prime}\right)$.

The design is such that it converts the bi-Gaussian beam with complex beam parameters $q_{0 x}$ and $q_{0 y}$ at the image source to that with complex beam parameters $q_{0 x^{\prime \prime}}$ and $q_{0 y^{\prime \prime}}$ at the beam waist according to equations (4) and (5). The values of $q_{0 x}$ and $q_{0 y}$ are obtained using equation (3) with $w_{0 x}=38.1 \mathrm{~mm}$ and $w_{0 y}=25.3 \mathrm{~mm}$, respectively. The values of $q_{0 x^{\prime \prime}}$ and $q_{0 y^{\prime \prime}}$ are also obtained using equation (3) with $w_{0 x^{\prime \prime}}=w_{0 y^{\prime \prime}}=w_{0}^{\prime \prime}$, respectively.

As can be seen from equation (2), we need two procedures to equalize the twodimensionally different complex parameters $\left(q_{0 x} \neq q_{0 y}\right)$ into the same value 
$\left(q_{0 x^{\prime \prime}}=q_{0 y^{\prime \prime}}\right)$, namely, the coincidence of the spot sizes and that of the curvature radii of the wave fronts. As can be seen from equations (4) and (5), the former consists of focusing and propagation, the latter consists of focusing only.

The mirror m1 plays the role of equalizing both spot sizes at the mirror m2. As can be seen from equation (2), this is expressed by

$$
\operatorname{Im}\left(\frac{1}{q_{2 x^{\prime}}}\right)=\operatorname{Im}\left(\frac{1}{q_{2 y^{\prime}}}\right)
$$

where $q_{2 x^{\prime}}$ and $q_{2 y^{\prime}}$ are the complex beam parameters in the $x^{\prime}$ - and $y^{\prime}$-directions at the mirror $\mathrm{m} 2$. If the focal length $f_{1 y}$ of mirror $\mathrm{ml}$ is given, the distance $d_{12}$ between the mirrors $\mathrm{m} 1$ and $\mathrm{m} 2$ is obtained by equation (6).

The value of $f_{1 y}$ can be determined by the condition that the beam focused by the mirror $m l$ has a waist size of $w_{0 y^{\prime \prime}}$. As can be seen from equations (4) and (5), they are given by

$$
\left.\begin{array}{l}
\frac{1}{q_{1} y^{\prime}}=\frac{1}{q_{1 y}}-\frac{1}{f_{1 y}} \\
q_{0 y^{\prime \prime}}=q_{y^{\prime}}+d^{\prime}
\end{array}\right\}
$$

where $q_{1 y}$ and $q_{1 y^{\prime}}$ are the complex beam parameters at mirror $\mathrm{ml}$ and just after reflection by mirror $\mathrm{ml}$, respectively and $d^{\prime}$ is the propagation distance from the mirror mil to the beam waist. If we notice that $q_{0 y^{\prime \prime}}$ is purely imaginary, $d^{\prime}$ is given by

$$
d^{\prime}=-\operatorname{Im}\left(q_{y^{\prime}}\right)
$$

Then, the distance $d^{\prime \prime}$ between the mirror $\mathrm{m} 2$ and the beam waist is given by

$$
d^{\prime \prime}=d^{\prime}-d_{12}
$$

Although the beam has a circular cross-section at the mirror $m 2$, the curvature radius of the wave front in the $x^{\prime \prime}$-direction is different from that in the $y^{\prime \prime}$-direction. The mirror $\mathrm{m} 2$ plays the role of equalizing both the curvature radii of the wave fronts. This is expressed by

$$
\frac{1}{q_{2 x^{\prime \prime}}}=\frac{1}{q_{2 x^{\prime}}}-\frac{1}{f_{2 x}}=\frac{1}{q_{2 y^{\prime}}}
$$

where $f_{2 x}$ is the focal length of mirror $\mathrm{m} 2$, and $q_{2 x^{\prime}}$ and $q_{2 y^{\prime}}$ are the complex beam parameters at the mirror $m 2$ and $q_{2 x^{\prime \prime}}$ is that just after reflection by the mirror $\mathrm{m} 2$.

\begin{tabular}{|c|c|c|c|c|c|c|c|c|c|c|}
\hline & \multicolumn{3}{|c|}{ Mirror ml } & \multicolumn{4}{|c|}{ Mirror m2 } & \multicolumn{3}{|c|}{ Beam waist } \\
\hline & $\begin{array}{c}f_{1 y} \\
(\mathrm{~mm})\end{array}$ & $\begin{array}{c}w_{x} \\
(\mathrm{~mm})\end{array}$ & $\begin{array}{c}w_{y} \\
(\mathrm{~mm})\end{array}$ & $\begin{array}{c}d_{12} \\
(\mathrm{~mm})\end{array}$ & $\begin{array}{c}f_{2 x} \\
(\mathrm{~mm})\end{array}$ & $\begin{array}{c}w_{x^{\prime}} \\
(\mathrm{mm})\end{array}$ & $\begin{array}{c}w_{y^{\prime}} \\
(\mathrm{mm})\end{array}$ & $\begin{array}{c}d^{\prime \prime} \\
(\mathrm{mm})\end{array}$ & $\begin{array}{l}W_{0 x^{\prime \prime}} \\
(\mathrm{mm})\end{array}$ & $\begin{array}{c}w_{0 y^{\prime \prime}} \\
(\mathrm{mm})\end{array}$ \\
\hline Case I & 1001 & 69.6 & 91.4 & 251.9 & 828 & 71.4 & 71.4 & 898.8 & 4.0 & 4.0 \\
\hline Case II & 2096 & 69.6 & 91.4 & 563.5 & 1909 & 73.6 & 73.6 & 2862 & 10.0 & 10.0 \\
\hline Case III & 3319 & 69.6 & 91.4 & 965.0 & 3395 & 76.5 & 76.5 & 5619 & 20.0 & 20.0 \\
\hline
\end{tabular}

Gaussian optics has been applied to the system, which produces beams with different waist sizes. Three cases are presented: Case I, $w_{0}^{\prime \prime}=4.0 \mathrm{~mm}$; Case II, $w_{0}^{\prime \prime}=10.0 \mathrm{~mm}$; and Case III, $w_{0}^{\prime \prime}=20.0 \mathrm{~mm}$. The results obtained by Gaussian optics are listed in table 1.

Table 1. The results obtained by Gaussian optics. $w_{x}$ and $w_{y}$ are the spotsizes in the $x$ - and $y$ directions, respectively. $w_{0 x^{\prime \prime}}$ and $w_{0 y^{\prime \prime}}$ are the waist sizes in the $x^{\prime \prime}$-and $y^{\prime \prime}$-directions, respectively. 


\section{Minrors m1 and $\mathrm{m} 2$}

The mirror $\mathrm{ml}$ is a parabolic cylinder whose focal axis is located in the $x^{\prime}-z^{\prime}$ plane (figure 4). If the rays parallel (opposite) to the $x^{\prime}$-axis are incident on the mirror at an angle of $\theta_{y}$, the rays reflected by the mirror $\mathrm{ml}$ intersect at the focal point $F_{1}$. In the present case, the mirror $m 1$ functions as a focusing element with the focal length of

$$
f_{1 y}=\frac{f_{y}}{\cos \theta_{y}}
$$

where $f_{y}(=707.6 \mathrm{~mm})$ is the focal length of the parabolic cylinder.

The mirror $\mathrm{m} 2$ is also a parabolic cylinder whose focal axis is located in the $y^{\prime \prime}-z^{\prime \prime}$ plane (figure 5). The mirror $m 2$ functions as a focusing element with the focal length of

$$
f_{2 x}=\frac{f_{x}}{\cos \theta_{x}}
$$

where $f_{x}(=585.5 \mathrm{~mm})$ is the focal length of the parabolic cylinder.

The angles $\theta^{\prime}$ and $\theta^{\prime \prime}$ in figure 2 are related to those $\theta_{y}$ and $\theta_{x}$, respectively. The relations are given by

$$
\left.\begin{array}{l}
\theta^{\prime}=\pi-2 \theta_{y} \\
\theta^{\prime \prime}=\pi-2 \theta_{x}
\end{array}\right\}
$$

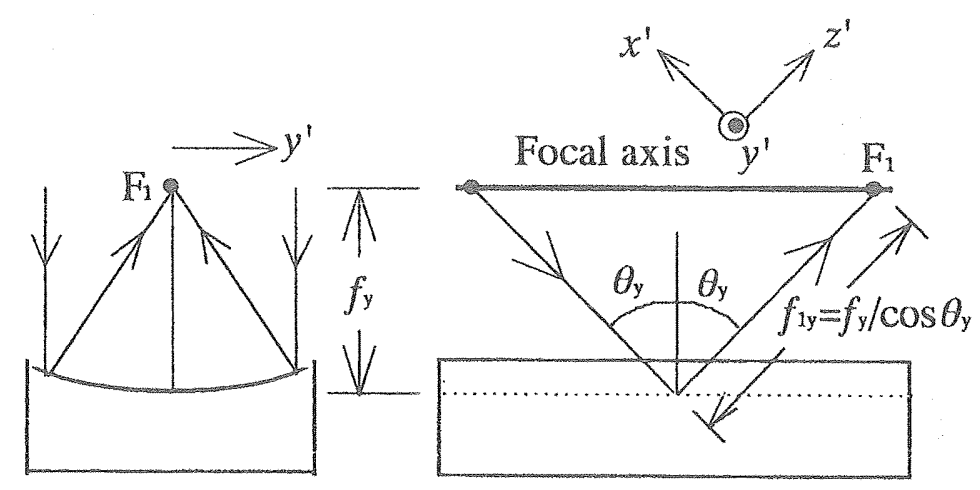

Figure 4. Geometry of the mirror $\mathrm{ml}$. The mirror is a parabolic cylinder with a focal length of $f_{y}$. Its focal axis is in the $x^{\prime}-z^{\prime}$ plane.
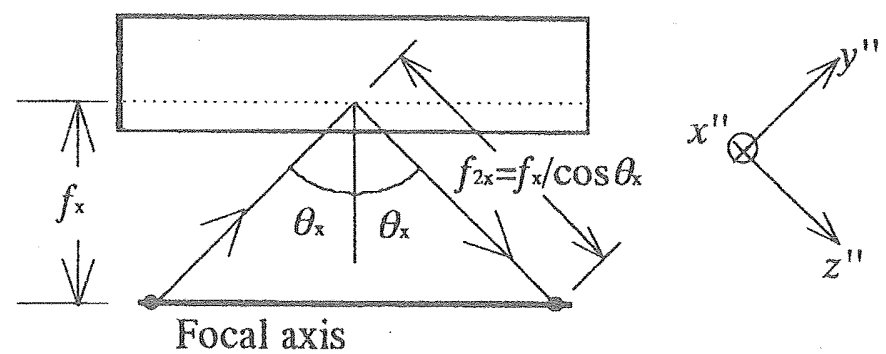

Figure 5. Geometry of the mirror $\mathrm{m} 2$. The mirror is a parabolic cylinder with the focal length of $f_{x}$. Its focal axis is in the $y^{\prime \prime}-z^{\prime \prime}$ plane. 
If necessary, problems due to changes of the system geometry can be avoided by introducing plane mirrors.

\section{Verification by the calculation using the Hruygens equation}

In order to verify the obtained results using Gaussian optics, we have carried out numerical calculations of the Huygens equation. At first, the incident electromagnetic fields at the surface of the mirror $\mathrm{ml}$ are calculated. The electromagnetic fields reflected by the mirror are obtained using the boundary conditions for a perfect conductor. The electromagnetic fields on the subsequent mirror $m 2$ are obtained by repeatedly using the Huygens equation and the boundary condition together with the previous calculated results as the sources.

For Case I $\left(w_{0}^{\prime \prime}=4.0 \mathrm{~mm}\right)$, calculated intensity contours are shown in figure 6 . The main beam with elliptical cross-section at mirror $m 1$ (figure 3 ) approaches to
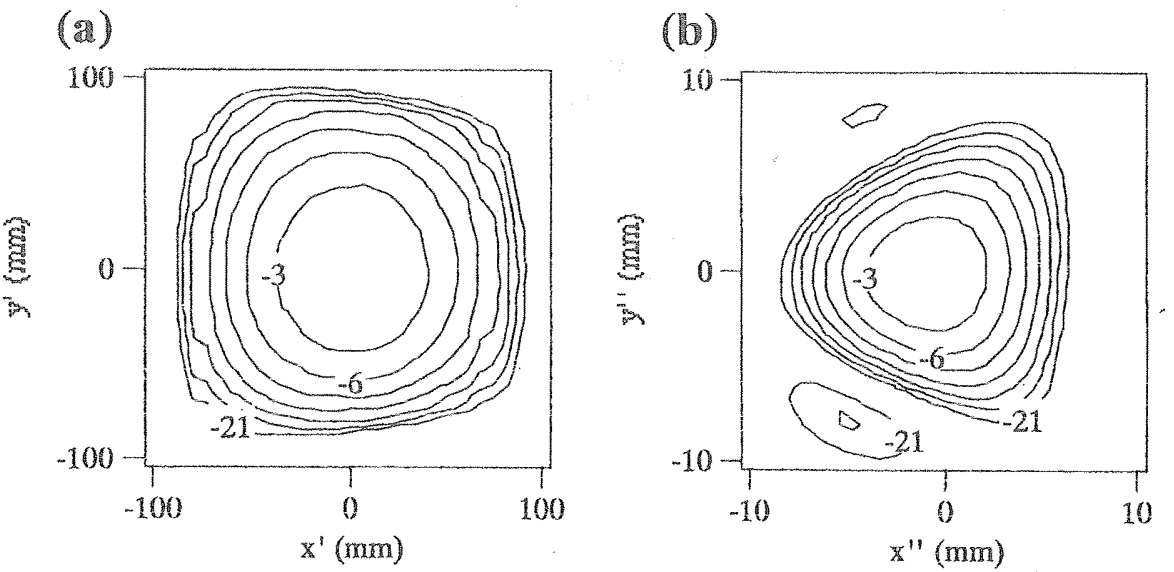

(c)

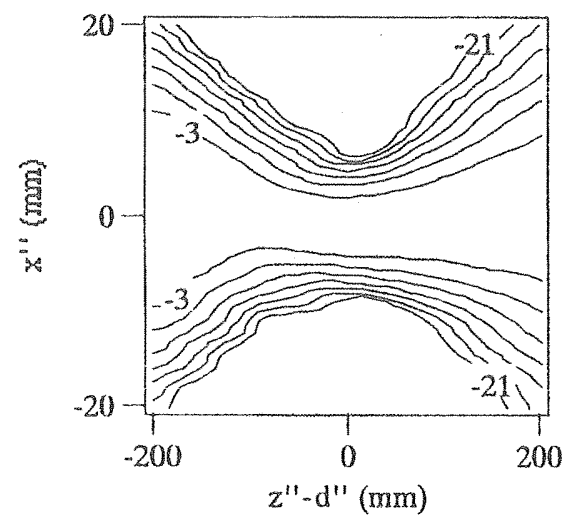

(d)

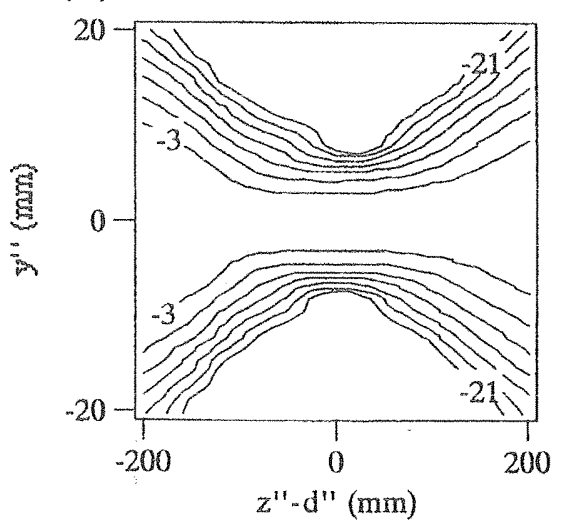

Figure 6. Calculated intensity contours corresponding to the Case $I\left(w_{0}^{\prime \prime}=4.0 \mathrm{~mm}\right)(\mathrm{a})$ at the mirror $\mathrm{m} 2$, (b) at the beam waist predicted by Gaussian optics. Contours are in decibels relative to the intensity maximum. (c) and (d) at the vicinity of the beam waist predicted by Gaussian optics. Contours are relative to the intensity along the $z^{\prime \prime}$-axis. 
almost circular at mirror $\mathrm{m} 2$ (figure $6(a)$ ) for the sake of the mirror $\mathrm{ml}$ and is wellcollimated at the beam waist as is predicted by Gaussian optics (figure $6(b)$ ). But, the beam has a triangular cross-section.

For Case II $\left(w_{0}^{\prime \prime}=10.0 \mathrm{~mm}\right)$ and Case III $\left(w_{0}^{\prime \prime}=20.0 \mathrm{~mm}\right)$, calculated intensity contours are shown in figure 7 and figure 8 , respectively. As the beam waist size is increased, the beam shape approaches to circular.

The beam produced by the image source contains sidelobes in addition to the main beam, as can be seen in figure 3. The quality of the beam is improved by truncating the side lobes by limiting the size of the mirror ml to the optimum size. This attempt is effective in removing sidelobes. In spite of the truncation of the sidelobes, most of power from the image source $(82.6 \%)$ is still reflected by the mirror m1.

Arrangements of the mirrors and results obtained by the calculations are listed in table 2. The results obtained by the Gaussian optics approach (table 1) are in good agreement with the calculations.

(\%)

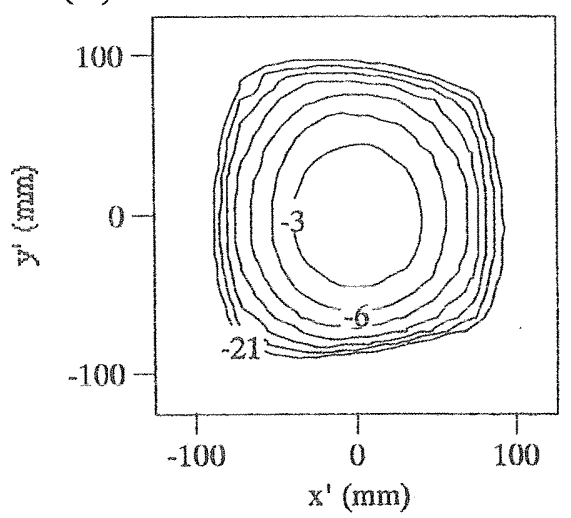

(c)

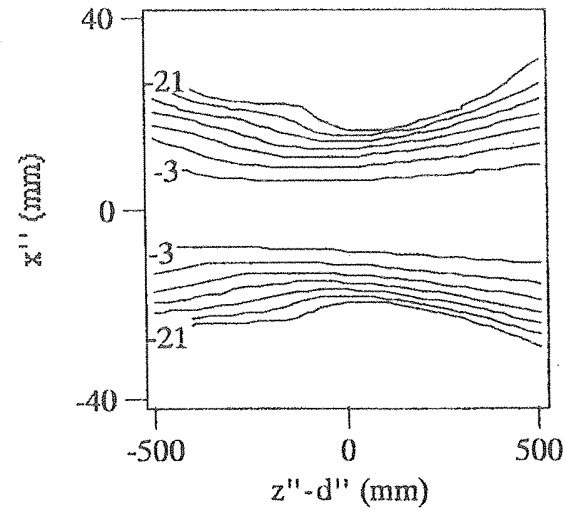

(b)

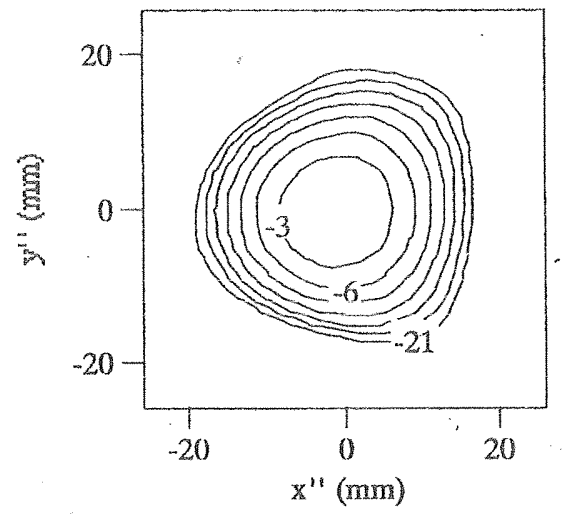

(d)

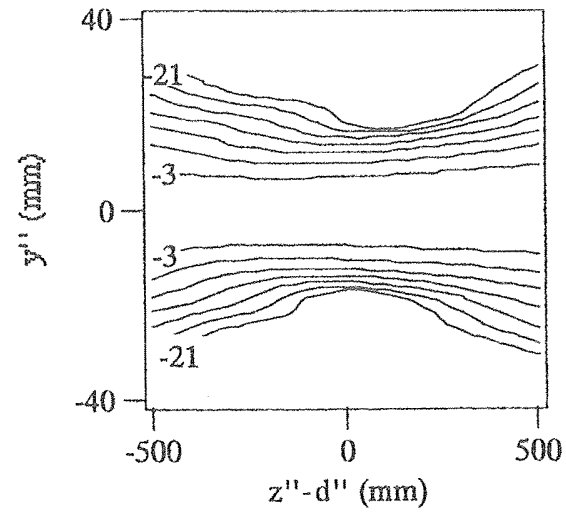

Figure 7. Calculated intensity contours corresponding to the Case $\Pi\left(w_{0}^{\prime \prime}=10.0 \mathrm{~mm}\right)$ (a) at the mirror $\mathrm{m} 2$, (b) at the beam waist predicted by Gaussian optics. Contours are in decibels relative to the intensity maximum. (c) and (d) at the vicinity of the beam waist predicted by Gaussian optics. Contours are relative to the intensity along the $z^{\prime \prime}$-axis. 
(a)

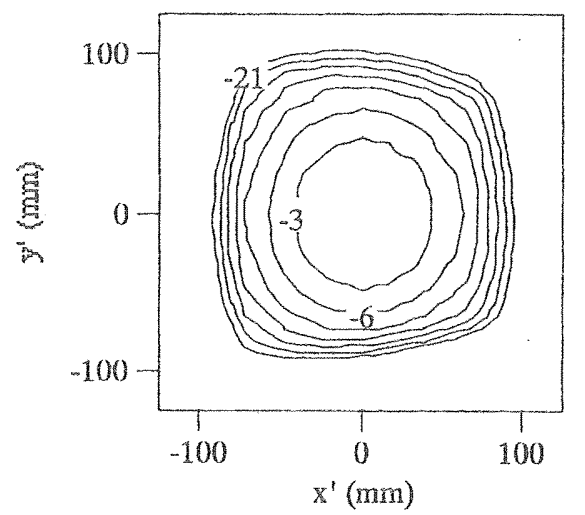

(c)

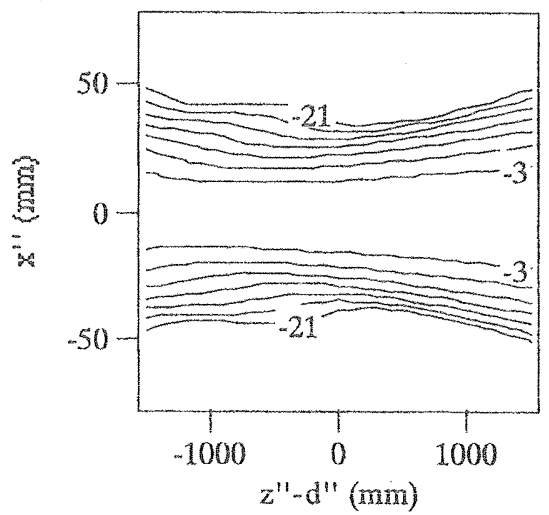

(b)

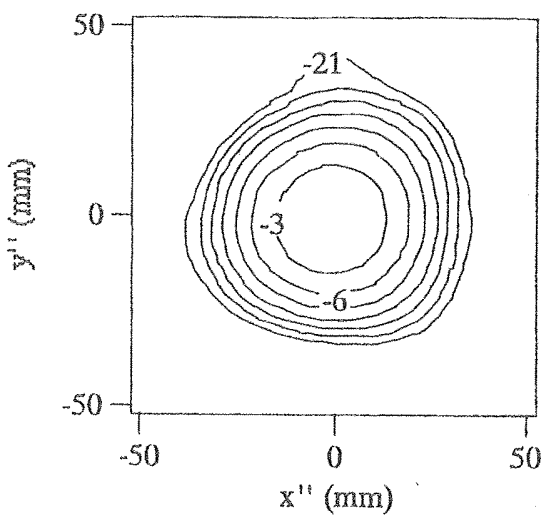

(d)

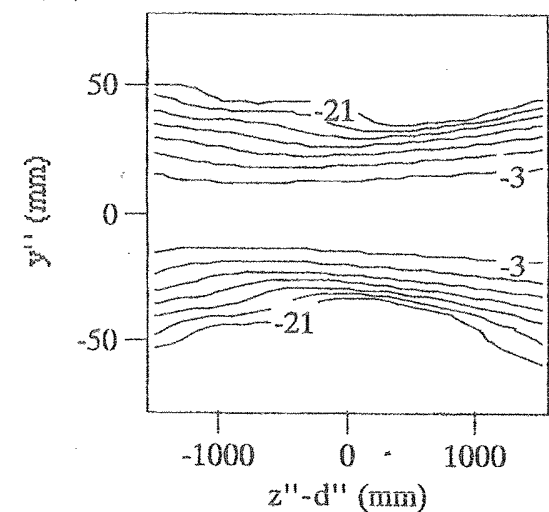

Figure 8. Calculated intensity contours corresponding to the Case $M r\left(w_{0}^{\prime \prime}=20.0 \mathrm{~mm}\right)(\mathrm{a})$ at the mirror $\mathrm{m} 2$, (b) at the beam waist predicted by Gaussian optics. Contours are in decibels relative to the intensity maximum. (c) and (d) at the vicinity of the beam waist. predicted by Gaussian optics. Contours are relative to the intensity along the $z^{\prime \prime}$-axis.

\begin{tabular}{|c|c|c|c|c|c|c|c|c|c|c|c|}
\hline & \multicolumn{3}{|c|}{$\begin{array}{c}\text { Mirror ml } \\
f_{y}=707.6(\mathrm{~mm}) \\
\end{array}$} & \multicolumn{4}{|c|}{$\begin{array}{c}\text { Mirror m2 } \\
f_{x}=585.5(\mathrm{~mm})\end{array}$} & \multicolumn{4}{|c|}{ Beam waist } \\
\hline & $\theta_{y}$ & $\begin{array}{c}w_{x} \\
(\mathrm{~mm})\end{array}$ & $\begin{array}{c}w_{y} \\
(\mathrm{~mm})\end{array}$ & $\begin{array}{c}d_{12} \\
(\mathrm{~mm})\end{array}$ & $\theta_{x}$ & $\begin{array}{c}w_{x^{\prime}} \\
(\mathrm{mm})\end{array}$ & $\begin{array}{c}w_{y^{\prime}} \\
(\mathrm{mm})\end{array}$ & $\begin{array}{c}d_{x^{\prime \prime}} \\
(\mathrm{mm})\end{array}$ & $\begin{array}{c}d_{y^{\prime \prime}} \\
(\mathrm{mm})\end{array}$ & $\begin{array}{l}w_{x^{\prime \prime}} \\
(\mathrm{mm})\end{array}$ & $\begin{array}{c}w_{y^{\prime \prime}} \\
(\mathrm{mm})\end{array}$ \\
\hline Case I & 45.0 & 69.6 & 91.4 & 251.9 & 45.0 & 63 & 69 & 891 & 899 & 5.0 & 5.1 \\
\hline Case II & 70.3 & 69.6 & 91.4 & 563.5 & 72.1 & 67 & 73 & 2751 & 2788 & 12.2 & 11.9 \\
\hline Case III & 77.7 & 69.6 & 91.4 & 965.0 & 80.1 & 72 & 76 & 5029 & 5211 & 23.7 & 23.3 \\
\hline
\end{tabular}

Table 2. Arrangement of the mirrors and results obtained by calculations using the Huygens equation. $w_{x}$ and $w_{y}$ are the spotsizes in the $x$-and $y$-directions, respectively. $d_{x^{\prime \prime}}$ are the distances between the mirror $\mathrm{m} 2$ and the waist, respectively. 


\section{Conclusion}

A system consisting of a quasi-optical antenna and two parabolic mirrors can convert the TE $\mathrm{TE}_{03}$ mode output $(f=301 \mathrm{GHz})$ of the Gyrotron FU IV into a well collimated, linearly polarized beam. This system offers the function to tune the size of the beam produced from $5 \mathrm{~mm}$ up to $24 \mathrm{~mm}$ by rotating and moving the two parabolic mirrors. The arrangement of the mirrors are obtained by using Gaussian optics.

For the sake of the beam quality, sidelobes of the beam produced by the quasioptical antenna are removed by locating the first mirror of suitable size far from the antenna. In spite of the truncation of the sidelobes, the beam produced by the system maintains most of power from the image source $(\sim 80 \%)$.

\section{Acknowledgments}

The authors would like to thank Dr D. Wagner, Universität Stuttgart, for valuable discussions and help.

This work was done as a collaboration between Fukui University, the National Institute for Fusion Science and the Universität Stuttgart, Germany. It has been supported by the Japan Society for the Promotion of Science (International Joint Research Projects).

The work of Fukui University was partially supported by a Grant-in-Aid from the Ministry of Education, Science and Culture of Japan.

Numerical calculations were made at the National Institute for Fusion Science Computer Center.

\section{References}

Brand, G. F., Fekete, P.W., Idehara, T., and Moore, K. J., 1990, Quasi-optical antennas for plasma scattering. International foumal of Electronics, 68, 1063-1073.

Fikete, P. W., Brand, G. F., and Ioemara, T., 1994, Scattering from discrete Alfven waves in a tokamak using a gyrotron radiation source. Plasma Physics and Controlled Nuclear Fusion, 36, 1407-1417.

Hong, K. D., Brand, G. F., and IDehara, T., 1993, A 150-600 GHz step-tunable gyrotron. Journal of Applied Physics, 74, 5250-5258.

Idehara, T., Shmmzu, Y., Ichikawa, K., Makino, S., Shibutani, K., Kurahashi, K. Tatsukama, T., OGawa, I., OKazaki, Y, and OKamoto, T., 1995, Development of a medium power, submillimeter wave gyrotron using a $17 \mathrm{~T}$ superconducting magnet. Physics of Plasmas, 2, 3246-3248.

Idemara, T., Yoshida, K., Nismma, N., Ogawa, I., Pereyaslavets, M. L., and Tatsukama, T., 1998, CW operation of a submillimeter wave gyrotron (Gyrotron FU IV) for high stability of the output frequency. International Journal of Infrared and Millimeter Waves, 19, 793-801.

Kawahata, K., Tetsuma, T., Fumta, J., Nagatsu, M., Omnish, H., Okajma, S., and IWASAKI, T., 1988, HCN laser scattering on the JIPP T-IIU tokamak. International Journal of Infrared and Millimeter Waves, 9, 655-665.

Morino, I., Fabian, M., Takeo, H., and Yamada, K., 1997, High-J rotational transitions of NNO measured with the NAIR terahertz spectrometer. Journal of Molecular Spectroscopy, 185, 142-146.

Omkubo, K., Kubo, S., Idei, H., Sato, M., Shmozuma, T., and Takmta, Y., 1997, Coupling of tilting Gaussian beam with hybrid mode in the corrugated waveguide. International Journal of Infrared and Millimeter Waves, 18, 23-41. 
Ogawa, I., Idemara, T., Pereyaslavets, M. L., and Kasparek, W., 1999 a, Design of the system converting a gyrotron output into a Gaussian-like beam, submitted to International Journal of Electronics.

OgaWa, I., Sakai, A., IDEhara, T, and Kasparek, W., 1997, A quasi-optical transmission line for plasma scattering measurements using a submillimeter wave gyrotron. International Joumal of Electronics, 83, 635-644.

Ogawa, I., Sakai, A., Idehara, T., and Kasparek, W., 1999 b, Application of the complex beam parameter to the design of quasi-optical transmission line for a submillimeter wave gyrotron. International Journal of Electronics, 86, 1071-1084.

Semet, A., MAse, A., Peebles, W. A., Lummann, N. C., and Zweben, JR., S., 1980, Study of low-frequency microturbulence in the Microtor tokamak by far-infrared laser scattering. Physical Review Letters, $45,445-448$

Spira-Hakkarainen, S., Kenneth, Kreischer, E., and Temkin, R. J., 1990, Submillimeter wave harmonic gyrotron experiment. IEEE Transactions on Plasma Science, 18, 334 342.

Suvorov, E. V., Holzhauer, E., Kasparex, W., Lubyako, L. V., Burov, A. B., Dryagin, Y. A., Filchenkov, S. E., Fraiman, A. A., Kukin, L. M., Kostrov, A. V., Rybdyx, D. A., Shtanyuk, A. M., Skalyga, N. K., Smolyakova, O. B., Erckmann, V., Geist, T. Kick, M., Lawua, H., and Rust, M., 1997, Collective Thomson scattering at WA-AS. Plasma Physics and Controlled Fusion, 39, B337-B351.

Tereumich, Y., Kubo, S., Ando, A., Yanagmoto, Y, OGura, K., Tanaka, H., Takahashi, J., Tonal, 1., Nakamura, M., Magxawa, T., Tanaka, S., and IDEHara, T., 1984, Study of low frequency density fluctuations in the WT-2 tokamak by MM and SUBMM wave scattering. Proceedings of 9th International Conference on Infrared and Millimeter Waves, Takarazuka, Japan, pp. 411-412.

VLASOV, S. N., and ORLova I. M., 1974, Quasioptical transformer which transforms the waves in a waveguide having a circular cross section into a highly directional wave beam. Radiofizika, 17, 115-119.

WADA, O., and NAKAJMA, M., 1986, Reffector antennas for electron cyclotron resonance heating of fusion plasma. Space Power, 6, 213-220.

Zaytsev, N. I.., Pankratova, T. B., Petelin, M. Y., and Flyagin, V. A., 1974, Millimeter- and submillimeter-wave gyrotrons. Radio Engineering Electronic Physics, 19, 103-107. 